\title{
Flexible multibody modelling for exact constraint design of compliant mechanisms
}

\author{
R.G.K.M. Aarts · J.P. Meijaard • J.B. Jonker
}

Received: 23 December 2010 / Accepted: 20 July 2011 / Published online: 5 November 2011

(C) The Author(s) 2011. This article is published with open access at Springerlink.com

\begin{abstract}
In high precision equipment, the use of compliant mechanisms is favourable as elastic joints offer the advantages of low friction and no backlash. If the constraints in a compliant mechanism are not carefully dealt with, even small misalignments can lead to changes in natural frequencies and stiffnesses. Such unwanted behaviour can be avoided by applying exact constraint design, which implies that the mechanism should have exactly the required degrees of freedom and non-redundant constraints so that the system is kinematically and statically determinate. For this purpose, we propose a kinematic analysis using a finite element based multibody modelling approach. In compliant mechanisms, the system's degrees of freedom are presented clearly from the analysis of a system in which the deformation modes with a low stiffness are free to deform while the deformation modes with a high stiffness are considered rigid. If the Jacobian matrix associated with the dependent coordinates is not full column or row rank, the system is under-constrained or over-constrained. The rank of this matrix is calculated from a singular value decomposition. For an under-constrained system, any motion in the mechanism that is not accounted for by the current set of degrees of freedom is visualised using data from the right singular matrix. For an over-constrained system, a statically indeterminate stress distribution is derived from the left singular matrix and is used to visualise the over-constraints. The analysis is exemplified for the design of a straight guiding mechanism, where under-constrained and over-constrained conditions are visualised clearly.
\end{abstract}

Keywords Exact constraint design - Kinematically indeterminate motion $\cdot$ Statically indeterminate stress distribution

\section{Introduction}

In high precision equipment, friction and backlash limit the achievable performance. Compliant mechanisms are mechanisms in which the mobility is realised from the deflection of

R.G.K.M. Aarts $(\bowtie) \cdot$ J.P. Meijaard · J.B. Jonker

Faculty of Engineering Technology, University of Twente, P.O. Box 217, 7500 AE Enschede,

The Netherlands

e-mail: R.G.K.M.Aarts@utwente.nl 
flexible elements rather than from kinematic joints. These elastic joints allow motion with almost zero friction and no backlash which is favourable for high accuracy. Flexible multibody modelling provides an efficient tool for analysing compliant mechanisms and can be utilised in the conceptual design phase of these mechanisms. At this phase, there is no need for very detailed and complex models that are time-consuming to analyse.

To obtain a predictable, deterministic behaviour, the principle of exact constraint design $[3,15]$ should be taken into consideration. In an over-constrained system even rather small misalignments may cause unwanted changes in natural frequencies and stiffnesses [13]. Recognising and repairing an under-constrained or over-constrained condition in a complicated system is not a trivial task. For this purpose, the designer can rely on his or her intuition or apply design rules and mathematical methods such as Grübler's mobility criterion for mechanisms [8], which is, e.g. discussed by Bottema [5]. Several authors provide a mathematical framework which is usually valid for a specific class of systems. Besseling [2] exploited the linear algebra theory of vector subspaces and matrices for the presentation of the classical linear theory of structures and proposed a framework for the assessment of kinematical and statical indeterminacy. Pellegrino and Calladine [14] study a "kinematic matrix" that relates small displacements of joints and elongations of connecting bars, and its transpose which is the "equilibrium matrix" relating the loads in the joints and the tension in the bars. Considering the null spaces of these matrices, they determine kinematically and statically indeterminate modes of pin-jointed assemblies. Similarly, Angeles and Gosselin [1] consider mechanisms with open and closed loop kinematic chains coupled by either revolute or prismatic pairs. The number of degrees of freedom of the chain is computed from the null space of a suitably defined Jacobian matrix.

Applying these analyses to compliant systems with elastic joints is not always straightforward. In this paper, we propose a flexible multibody modelling approach which is implemented in the SPACAR software package [10]. This flexible multibody approach is based on a non-linear finite element method, in which for each element a fixed number of deformation modes are defined. Typical flexible members of compliant mechanisms are wire and sheets flexures. These flexures may be considered as one-dimensional structures which can be correctly modelled by beams. Due to the definition of physically meaningful deformation modes and the sound inclusion of the non-linear geometrical effects at the element level [11], only a rather small number of elastic beam elements are needed to model typical elastic components accurately. In the kinematic analysis, the nodal coordinates and deformation mode coordinates are explicitly classified into constrained, dependent, and independent coordinates and deformations. The stiffnesses of the flexures are such that they allow motion in directions with low stiffness whereas the motion is constrained in directions with a relatively large stiffness. For the kinematic analysis, this is taken into account by considering the deformation modes with a high stiffness to be rigid, i.e. having constrained deformation mode coordinates. The deformation modes with low stiffnesses are allowed to deform, so the associated deformation mode coordinates are dependent or independent. Then it appears that in order to satisfy exact constraint design the Jacobian matrix associated with the dependent coordinates must be square and full rank: otherwise the system is under-constrained and/or over-constrained. Singular value decomposition [7] is an elegant mathematical and numerical technique to compute the rank of a matrix.

The modelling approach is briefly summarised in the next section. In Sect. 3, it is outlined how over-constrained and under-constrained systems can be analysed. This procedure is applied to the example of a straight guiding mechanism in Sect. 4. Exact constraint designs are verified with kinematic analyses. Section 5 summarises the conclusions. 


\section{Finite element modelling}

\subsection{Spatial finite elements}

In this paper, compliant mechanisms are modelled with a flexible multibody system approach. The formulation is based on a non-linear finite element approach. In this finite element approach, the multibody system is modelled as an assembly of rigid body structures interconnected through a variety of connections such as flexible hinges and beams. The location of each element $k$ is described relative to a fixed inertial coordinate system by a set of nodal coordinates $\mathbf{x}^{(k)}$, valid for large displacements and rotations. Translational and rotational coordinates are used to describe the positions of the end nodes and the orientation of orthogonal base vectors or triads, rigidly attached to the element nodes.

Essential is the definition of physically meaningful deformation modes of the element. The deformation modes are specified by a vector of discrete deformations $\boldsymbol{\varepsilon}^{(k)}$ that are invariant for rigid body motions of the element [9]. The number of deformations is equal to the number of nodal coordinates minus the number of degrees of freedom of the element as a rigid body. The deformations are explicitly described as non-linear deformation functions of the nodal coordinates

$$
\boldsymbol{\varepsilon}^{(k)}=\mathscr{D}^{(k)}\left(\mathbf{x}^{(k)}\right)
$$

A rather simple spatial element is the (slider) truss element, which represents a (straight) connection between two nodes $p$ and $q$ whose position is described by the Cartesian position vectors $\mathbf{x}^{p}$ and $\mathbf{x}^{q}$. A rotation about the element axis $p q$ does not appear in the description of the element position. Hence, the element has six nodal coordinates and five rigid body degrees of freedom, giving rise to a single deformation mode $\varepsilon_{1}^{(k)}$, associated with the elongation of the element, i.e.

$$
\varepsilon_{1}^{(k)}=\mathscr{D}_{1}^{(k)}\left(\mathbf{x}^{(k)}\right)=l^{(k)}-l_{0}^{(k)},
$$

where $l^{(k)}=\left\|\mathbf{x}^{q}-\mathbf{x}^{p}\right\|$ is the distance between the nodal points $\mathbf{x}^{p}$ and $\mathbf{x}^{q}$ and $l_{0}^{(k)}$ is the reference length of the element.

To model the wire and sheet flexures that are often used in compliant mechanisms, the spatial beam element is well-suited. The location of this element is described by the positions of the end nodes $p$ and $q$, as well as their orientations. Then there are twelve independent nodal coordinates and six rigid body degrees of freedom, so that six independent deformation modes can be defined. For the spatial flexible beam, one deformation mode $\varepsilon_{1}$ is taken to describe the elongation, $\varepsilon_{2}$ for torsion, and four modes $\varepsilon_{3-6}$ for the bending deformations of the element. For readability, the superscripts $(k)$ indicating the element number are omitted. Expressions for these deformation modes are [11, 13]:

$$
\begin{aligned}
& \varepsilon_{1}=\bar{\varepsilon}_{1}+\left(2 \bar{\varepsilon}_{3}^{2}+\bar{\varepsilon}_{3} \bar{\varepsilon}_{4}+2 \bar{\varepsilon}_{4}^{2}+2 \bar{\varepsilon}_{5}^{2}+\bar{\varepsilon}_{5} \bar{\varepsilon}_{6}+2 \bar{\varepsilon}_{6}^{2}\right) /\left(30 l_{0}\right)+c_{t} \bar{\varepsilon}_{2}^{2} /\left(2 l_{0}^{3}\right), \quad \text { (elongation) } \\
& \varepsilon_{2}=\bar{\varepsilon}_{2}+\left(-\bar{\varepsilon}_{3} \bar{\varepsilon}_{6}+\bar{\varepsilon}_{4} \bar{\varepsilon}_{5}\right) / l_{0}, \quad \text { (torsion) } \\
& \varepsilon_{3}=\bar{\varepsilon}_{3}+\bar{\varepsilon}_{2}\left(\bar{\varepsilon}_{5}+\bar{\varepsilon}_{6}\right) /\left(6 l_{0}\right), \quad \text { (bending in } x z \text {-plane) } \\
& \varepsilon_{4}=\bar{\varepsilon}_{4}-\bar{\varepsilon}_{2}\left(\bar{\varepsilon}_{5}+\bar{\varepsilon}_{6}\right) /\left(6 l_{0}\right), \\
& \varepsilon_{5}=\bar{\varepsilon}_{5}-\bar{\varepsilon}_{2}\left(\bar{\varepsilon}_{3}+\bar{\varepsilon}_{4}\right) /\left(6 l_{0}\right), \quad \text { (bending in } x y \text {-plane) } \\
& \varepsilon_{6}=\bar{\varepsilon}_{6}+\bar{\varepsilon}_{2}\left(\bar{\varepsilon}_{3}+\bar{\varepsilon}_{4}\right) /\left(6 l_{0}\right), \quad
\end{aligned}
$$



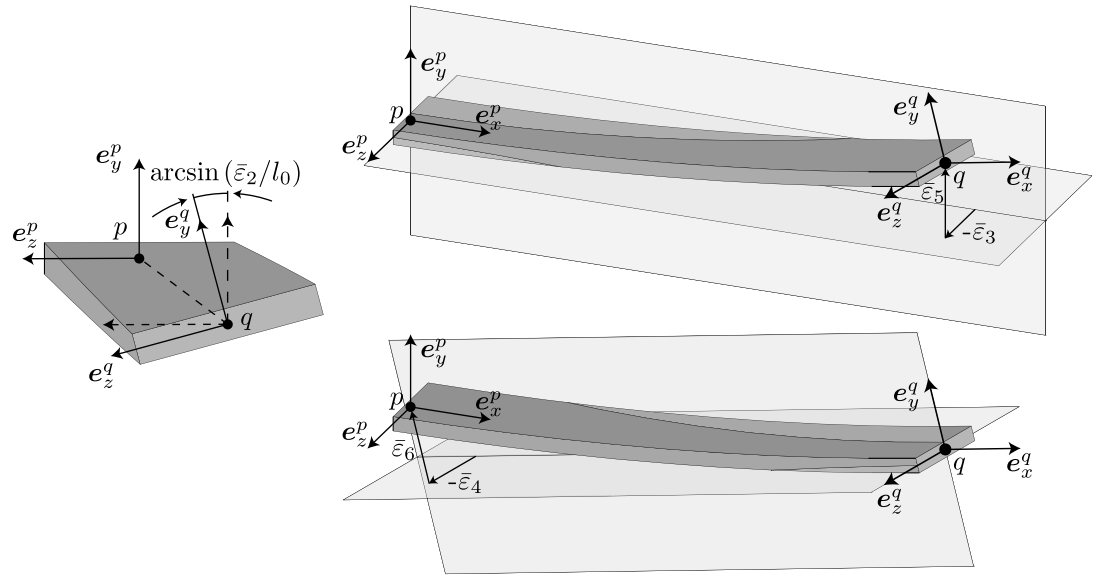

Fig. 1 Deformations $\varepsilon_{2}-\varepsilon_{6}$ of the spatial beam element (reprinted from [4])

with

$$
\begin{aligned}
& \bar{\varepsilon}_{1}=l-l_{0}, \\
& \bar{\varepsilon}_{2}=l_{0}\left(\mathbf{e}_{z}^{p} \cdot \mathbf{e}_{y}^{q}-\mathbf{e}_{y}^{p} \cdot \mathbf{e}_{z}^{q}\right) / 2, \\
& \bar{\varepsilon}_{3}=-l_{0} \mathbf{e}_{l} \cdot \mathbf{e}_{z}^{p}, \\
& \bar{\varepsilon}_{4}=l_{0} \mathbf{e}_{l} \cdot \mathbf{e}_{z}^{q}, \\
& \bar{\varepsilon}_{5}=l_{0} \mathbf{e}_{l} \cdot \mathbf{e}_{y}^{p}, \\
& \bar{\varepsilon}_{6}=-l_{0} \mathbf{e}_{l} \cdot \mathbf{e}_{y}^{q},
\end{aligned}
$$

where $l=\left\|\mathbf{x}^{q}-\mathbf{x}^{p}\right\|$ is again the distance between the nodal points $\mathbf{x}^{p}$ and $\mathbf{x}^{q}, l_{0}$ is the reference length of the element and $\mathbf{e}_{l}=\left(\mathbf{x}^{q}-\mathbf{x}^{p}\right) / l$ is the unit vector directed from node $p$ to node $q$. The term with the torsional constant $c_{t}$ accounts for torsion-elongation coupling [13]. Figure 1 illustrates five of these deformation modes and most of the unit vectors in the expressions. The elements account for geometrically non-linear effects due to interaction between deformation modes. Consequently, the expression for the beam elongation $\varepsilon_{1}$ is more complicated than (2) for the truss elements, as additional terms take into account the additional elongation of the beam axis caused by torsion and bending. The additional terms in $\varepsilon_{2}$ measure extra torsion of the beam caused by its bending. Furthermore, the additional terms in the expressions for the bending deformations account for the effect of asymmetrical bending caused by a twist of the beam. Consequently, accurate models can be obtained with a relatively small numbers of elements even for the case when large deflections are considered $[11,13]$.

A single beam element as visualised in Fig. 1 is well-suited to model a sheet flexure, which is relatively thin in one direction, i.e. the $y$-direction in the figure. When used in a compliant mechanism, the sheet flexure is meant to deform relatively easily by out-ofplane bending and torsion, i.e. the deformations $\varepsilon_{5,6}$ and $\varepsilon_{2}$ for this beam element. It should provide support in other directions as the stiffnesses for in-plane bending and elongations are much higher, i.e. for the deformations $\varepsilon_{3,4}$ and $\varepsilon_{1}$. Clearly, the definitions of the deformation modes match perfectly with the deformations that are relevant to describe the behaviour of the sheet flexure. This is also true for a wire flexure, which is thin in two directions. It will deform easily in torsion and all bending directions $\left(\varepsilon_{2-6}\right)$, while only the stiffness for the elongation $\left(\varepsilon_{1}\right)$ is high. 


\subsection{Kinematic analysis and the degrees of freedom}

For the entire multibody system, the assembly of finite elements is realised by defining a global vector $\mathbf{x}$ of all nodal coordinates. The discrete deformation functions of the elements constituting the multibody system can then be described in terms of the components of vector $\varepsilon$ yielding the non-linear vector function

$$
\varepsilon=\mathscr{D}(\mathbf{x})
$$

which represents the basic equations for the kinematic analysis. Kinematic constraints can be introduced by putting conditions on the nodal coordinates, denoted with $\mathbf{x}^{(o)}$ for support coordinates, as well as by prescribing the deformations $\boldsymbol{\varepsilon}^{(o)}$ to be zero for rigid bodies. In this paper, all kinematic constraints are assumed to be holonomic.

An important notion in the kinematic and dynamic analysis of mechanical systems is that of degrees of freedom (DOFs). The number of kinematic degrees of freedom is the smallest number of coordinates $n_{\text {dof }}$ that describe, together with the fixed, time-independent kinematic constraints, the configuration of the multibody system in a continuous way. We call them independent or generalised coordinates $\mathbf{q}$ which can be either absolute generalised coordinates, denoted $\mathbf{x}^{(m)}$, as well as relative generalised coordinates, denoted $\boldsymbol{\varepsilon}^{(m)}$, i.e.

$$
\mathbf{q}=\left[\begin{array}{l}
\mathbf{x}^{(m)} \\
\boldsymbol{\varepsilon}^{(m)}
\end{array}\right] .
$$

In accordance with the above specified constraints and the choice of generalised coordinates, the vectors $\mathbf{x}$ and $\boldsymbol{\varepsilon}$ can now be partitioned as

$$
\mathbf{x}=\left[\begin{array}{l}
\mathbf{x}^{(o)} \\
\mathbf{x}^{(c)} \\
\mathbf{x}^{(m)}
\end{array}\right] \quad \text { and } \quad \boldsymbol{\varepsilon}=\left[\begin{array}{l}
\boldsymbol{\varepsilon}^{(o)} \\
\boldsymbol{\varepsilon}^{(m)} \\
\boldsymbol{\varepsilon}^{(c)}
\end{array}\right],
$$

where the superscript $o$ denotes invariant nodal coordinates or deformations having a fixed prescribed value, the superscript $c$ denotes dependent nodal coordinates or deformations and the superscript $m$ denotes independent (or generalised) nodal coordinates or deformations.

By differentiating (5) to time and using the chain rule, it appears that the velocities $\dot{\mathbf{x}}$ and $\dot{\varepsilon}$ must obey

$$
\dot{\varepsilon}=\mathscr{D}_{, \mathrm{x}} \dot{\mathbf{x}},
$$

where the notation $\mathscr{D}_{, \mathbf{x}}$ indicates partial differentiation of the deformation functions with respect to the nodal coordinates $\mathbf{x}$.

\subsection{Equilibrium equations}

Forces $\mathbf{f}$ can be applied to the nodal points and are dual to the velocities $\dot{\mathbf{x}}$. Generalised stress resultants represent the loading state of each element and are assembled in the vector $\sigma$. These generalised stress resultants are dual to $\dot{\varepsilon}$. The unknown stress resultants and reaction forces are computed from the equilibrium equations [12]

$$
\mathscr{D}_{, \mathbf{x}}^{T} \boldsymbol{\sigma}=\mathbf{f} \text {. }
$$




\section{Over-constrained and under-constrained systems}

In the previous section, it was assumed that the $n_{\text {dof }}$ independent or generalised coordinates describe, together with the constraints, the configuration of the system. This aspect will be addressed in more detail next.

\subsection{Kinematically indeterminate or under-constrained system}

With the partitioning of (7) for $\mathbf{x}$ and $\boldsymbol{\varepsilon},(8)$ for the velocities can be written as

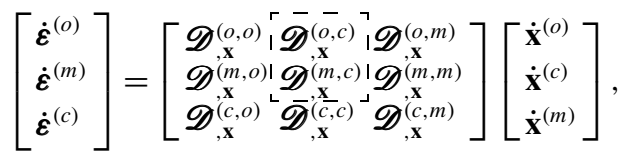

in which the derivative function $\mathscr{D}_{, \mathbf{x}}$ is split in components where subsets of the deformation functions are differentiated with respect to a part of the nodal coordinates. The indicated submatrix

$$
\mathbf{D}^{(c)}=\left[\begin{array}{c}
\mathscr{D}_{, \mathbf{x}}^{(o, c)} \\
\mathscr{D}_{, \mathbf{x}}^{(m, c)}
\end{array}\right]
$$

is the Jacobian matrix associated with the dependent coordinates $\mathbf{x}^{(c)}$. It relates the velocities of the dependent coordinates $\dot{\mathbf{x}}^{(c)}$ with the constrained deformations $\dot{\boldsymbol{\varepsilon}}^{(o)}$ and velocities of the generalised coordinates $\dot{\boldsymbol{\varepsilon}}^{(m)}, \dot{\mathbf{x}}^{(m)}$ as

$$
\mathbf{D}^{(c)} \dot{\mathbf{x}}^{(c)}=\left[\begin{array}{l}
\dot{\boldsymbol{\varepsilon}}^{(o)} \\
\dot{\boldsymbol{\varepsilon}}^{(m)}
\end{array}\right]-\left[\begin{array}{l}
\mathscr{D}_{, \mathbf{x}}^{(o, m)} \\
\mathscr{D}_{, \mathbf{x}}^{(m, m)}
\end{array}\right] \dot{\mathbf{x}}^{(m)},
$$

where the term with $\dot{\mathbf{x}}^{(o)}=\mathbf{0}$ has been omitted. If the inverse of the Jacobian matrix $\mathbf{D}^{(c)}$ exists, the velocities $\dot{\mathbf{x}}^{(c)}$ can be computed from the constraints $\dot{\boldsymbol{\varepsilon}}^{(o)}=\mathbf{0}$ and the velocities of the generalised coordinates $\dot{\boldsymbol{\varepsilon}}^{(m)}, \dot{\mathbf{x}}^{(m)}$. For the existence of this inverse, the Jacobian matrix must be square. This implies that the dimension of $\mathbf{x}^{(c)}$ is equal to the sum of the dimensions of $\boldsymbol{\varepsilon}^{(o)}$ and $\boldsymbol{\varepsilon}^{(m)}$, so

$$
n_{x}^{(c)}=n_{\varepsilon}^{(o)}+n_{\varepsilon}^{(m)},
$$

where $n_{x}^{(c)}$ is the number of dependent coordinates $\mathbf{x}^{(c)}$ and $n_{\varepsilon}^{(o)}, n_{\varepsilon}^{(m)}$ are the number of constrained and independent deformation modes, respectively. The number of dependent coordinates $n_{x}^{(c)}$ is the total number of coordinates $n_{x}$ minus the numbers of constrained coordinates $n_{x}^{(o)}$ and independent generalised nodal coordinates $n_{x}^{(m)}$ :

$$
n_{x}^{(c)}=n_{x}-\left(n_{x}^{(o)}+n_{x}^{(m)}\right) .
$$

Then it follows that $\mathbf{D}^{(c)}$ is square if the number of all kinematic degrees of freedom $n_{\text {dof }}=$ $n_{x}^{(m)}+n_{\varepsilon}^{(m)}$ satisfies

$$
n_{\text {dof }}=n_{x}-\left(n_{x}^{(o)}+n_{\varepsilon}^{(o)}\right),
$$

so the number of degrees of freedom equals the number of nodal coordinates $\mathbf{x}$ minus the number of absolute and holonomic constraints, $\mathbf{x}^{(o)}$ and $\boldsymbol{\varepsilon}^{(o)}$. This is in agreement with, e.g. Grübler's mobility criterion for mechanisms [5].

Equation (15) is a necessary condition for the existence of $\mathbf{D}^{(c)-1}$ as it results in a square matrix $\mathbf{D}^{(c)}$. It is not a sufficient condition as $\mathbf{D}^{(c)}$ must also be regular, or equivalently 
the matrix $\mathbf{D}^{(c)}$ should be full rank. For any square or rectangular matrix, the rank can be determined from its singular value decomposition which for $\mathbf{D}^{(c)}$ can be written as

$$
\mathbf{D}^{(c)}=\mathbf{U} \boldsymbol{\Sigma} \mathbf{V}^{T}
$$

in which $\mathbf{U}$ is an orthogonal $m \times m$ matrix, $\mathbf{V}$ is an orthogonal $n \times n$ matrix and matrix $\boldsymbol{\Sigma}$ is an $m \times n$ diagonal matrix with non-negative real numbers on the diagonal, denoting $m$ the number of rows in $\mathbf{D}^{(c)}$ and $n$ the number of columns. The inverse of $\mathbf{D}^{(c)}$ exists if the matrix is square, i.e. $m=n$, and all singular values are positive.

If some of the singular values are zero, or more generally, if $\mathbf{D}^{(c)}$ is not full column rank, it can be seen from (12) that there is a non-zero solution for $\dot{\mathbf{x}}^{(c)}$ that satisfies the constraint $\dot{\boldsymbol{\varepsilon}}^{(o)}=\mathbf{0}$ while the velocities of the generalised coordinates $\dot{\boldsymbol{\varepsilon}}^{(m)}, \dot{\mathbf{x}}^{(m)}$ are also zero. That means the system is kinematically indeterminate or under-constrained. Matrix $\mathbf{D}^{(c)}$ is column rank deficient if there are more columns than rows, i.e. $n>m$. In addition, zero singular values increase the column rank deficiency. By combining the singular value decomposition (16) with (10), it can be seen that each column in $\mathbf{V}$ accompanying one of the zero singular values and the columns that are in excess of the rows in $\mathbf{D}^{(c)}$ specify a vector of velocities $\dot{\mathbf{x}}^{(c)}$ which represents the motion of a kinematically indeterminate mode.

\subsection{Statically indeterminate or over-constrained system}

For the solution of the equilibrium equations (9), the nodal force vector $\mathbf{f}$ and the vector of generalised stress resultants $\sigma$ are also partitioned in accordance with (7) as

$$
\mathbf{f}=\left[\begin{array}{c}
\mathbf{f}^{(o)} \\
\mathbf{f}^{(c)} \\
\mathbf{f}^{(m)}
\end{array}\right] \quad \text { and } \quad \boldsymbol{\sigma}=\left[\begin{array}{c}
\boldsymbol{\sigma}^{(o)} \\
\boldsymbol{\sigma}^{(m)} \\
\boldsymbol{\sigma}^{(c)}
\end{array}\right] .
$$

With this partitioning (9) is written as

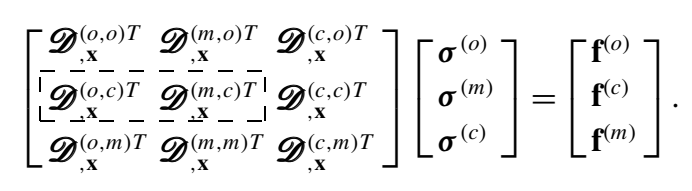

The matrix in the left-hand side is the transpose of the derivative function $\mathscr{D}_{, \mathbf{x}}$ in (10). Hence, the transpose $\mathbf{D}^{(c) T}$ can also be recognised as the indicated submatrix which gives an expression for the generalised stress resultants $\boldsymbol{\sigma}^{(o)}$ and $\boldsymbol{\sigma}^{(m)}$ as

$$
\mathbf{D}^{(c) T}\left[\begin{array}{l}
\boldsymbol{\sigma}^{(o)} \\
\boldsymbol{\sigma}^{(m)}
\end{array}\right]=\mathbf{f}^{(c)}-\mathscr{D}_{, \mathbf{x}}^{(c, c) T} \boldsymbol{\sigma}^{(c)} .
$$

If the inverse of $\mathbf{D}^{(c)}$ exists, then also the inverse of its transpose $\mathbf{D}^{(c) T}$ exists and the generalised stress resultants $\boldsymbol{\sigma}^{(o)}$ and $\boldsymbol{\sigma}^{(m)}$ that are dual to the relative constraints $\boldsymbol{\varepsilon}^{(o)}$ and independent deformation mode coordinates $\boldsymbol{\varepsilon}^{(m)}$ can be computed from the nodal forces and the other generalised stress resultants in the right-hand side of (19). In the case $\mathbf{D}^{(c) T}$ is not full column rank, a non-zero solution of the generalised stress resultants $\boldsymbol{\sigma}^{(o)}$ and $\boldsymbol{\sigma}^{(m)}$ exists for which the nodal forces $\mathbf{f}^{(c)}$ and generalised stress resultants $\boldsymbol{\sigma}^{(c)}$ are zero. This indicates a statically indeterminate or over-constrained system. 
Table 1 System properties depending on the number of rows $m$, the number of columns $n$, and the rank of matrix $\mathbf{D}^{(c)}$

\begin{tabular}{llll}
\hline$m=n$ & $\operatorname{rank}\left(\mathbf{D}^{(c)}\right)=m=n$ & statically determinate & kinematically determinate \\
& $\operatorname{rank}\left(\mathbf{D}^{(c)}\right)<m=n$ & statically indeterminate & kinematically indeterminate \\
\hline$m>n$ & $\operatorname{rank}\left(\mathbf{D}^{(c)}\right)=n<m$ & statically indeterminate & kinematically determinate \\
& $\operatorname{rank}\left(\mathbf{D}^{(c)}\right)<n<m$ & statically indeterminate & kinematically indeterminate \\
\hline$m<n$ & $\operatorname{rank}\left(\mathbf{D}^{(c)}\right)=m<n$ & statically determinate & kinematically indeterminate \\
& $\operatorname{rank}\left(\mathbf{D}^{(c)}\right)<m<n$ & statically indeterminate & kinematically indeterminate \\
\hline
\end{tabular}

Note that the column rank of $\mathbf{D}^{(c) T}$ equals the row rank of $\mathbf{D}^{(c)}$. Matrix $\mathbf{D}^{(c)}$ is row deficient if there are more rows than columns, i.e. $m>n$, or there are zero singular values. From (16), the singular value decomposition of $\mathbf{D}^{(c) T}$ follows immediately as

$$
\mathbf{D}^{(c) T}=\mathbf{V} \Sigma^{T} \mathbf{U}^{T} .
$$

By combining this singular value decomposition with (18), we recognise that each column in $\mathbf{U}$ accompanying one of the zero singular values and the columns that are in excess of the rows in $\mathbf{D}^{(c) T}$ are a non-zero solution of the generalised stress resultants $\boldsymbol{\sigma}^{(o)}$ and $\boldsymbol{\sigma}^{(m)}$ that represents a set of statically indeterminate stress resultants.

\subsection{Kinematic analysis and visualisation}

In Table 1, it is summarised that row and/or column rank deficiency of matrix $\mathbf{D}^{(c)}$ implies a statically and/or kinematically indeterminate system. Moreover, with each excess row or column and with each zero singular value there is a column in the matrix $\mathbf{U}$ or $\mathbf{V}$ that describes the indeterminate mode. The kinematically indeterminate modes are found in the right singular matrix $\mathbf{V}$ and can directly be used to visualise a motion of the system, i.e. the motion resulting from a non-zero velocity $\dot{\mathbf{x}}^{(c)}$. The statically indeterminate modes are found in the left singular matrix $\mathbf{U}$ and give non-zero generalised stress resultants $\boldsymbol{\sigma}^{(o)}, \boldsymbol{\sigma}^{(m)}$. Boer et al. [4] have shown how the stress distribution in the elements can be computed from the generalised stress resultants. This analysis is well-suited to visualise the statically indeterminate stress distribution that results from the singular value decomposition.

To illustrate this approach, we consider the system with twelve rigid trusses shown in Fig. 2 [14]. Eight trusses $1-8$ are supported in four support nodes A $(-1,-1,0)$, $\mathrm{B}(1,-1,0), \mathrm{C}(1,1,0)$, and $\mathrm{D}(-1,1,0)$ in the $z=0$ plane. They are interconnected in the $z=1$ plane in the nodal points $\mathrm{E}(0,-1,1), \mathrm{F}(1,0,1), \mathrm{G}(0,1,1)$, and $\mathrm{H}(-1,0,1)$. Four trusses 9-12 connect these points also directly as shown in the figure. In the eight support and connection points, the trusses can rotate freely. As pointed out in Sect. 2.1, each truss element connects two Cartesian nodal points and has only one deformation which is its elongation $\varepsilon_{1}^{(k)}$ according to (2). Only Cartesian coordinates are needed to analyse this system. There are $n_{x}^{(o)}=4 \times 3=12$ support coordinates $\mathbf{x}^{(o)}$ (in the $z=0$ plane). The nodes in the $z=1$ plane give $n_{x}^{(c)}=4 \times 3=12$ dependent coordinates $\mathbf{x}^{(c)}$. There are $n_{\varepsilon}^{(o)}=12$ constrained deformations $\boldsymbol{\varepsilon}^{(o)}$ counting the elongations $\varepsilon_{1}^{(1)}-\varepsilon_{1}^{(12)}$ in all trusses. Hence, $\mathbf{D}^{(c)}$ is a square $12 \times 12$ matrix. According to (15), the number of degrees of freedom $n_{\mathrm{dof}}$ is zero, suggesting that the system is a structure and should not move.

For a singular value analysis of matrix $\mathbf{D}^{(c)}$, we consider the definitions of the coordinates and deformations of which the time derivatives appear in (12) in somewhat more detail. No independent deformations and coordinates are defined, so the vectors $\boldsymbol{\varepsilon}^{(m)}$ and 


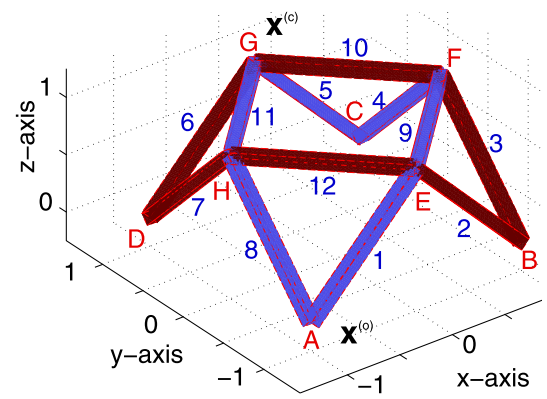

(a) statically indeterminate stress distribution (colours indicate sign of the stresses)

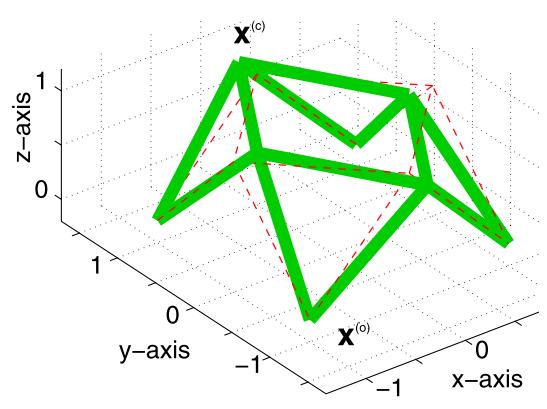

(b) kinematically indeterminate motion (dashes show the initial configuration)

Fig. 2 System with 12 rigid trusses, supported in the nodes $\mathbf{x}^{(o)}(z=0$ plane) and connected in the nodes $\mathbf{x}^{(c)}(z=1$ plane). In (a), the numbers $1-12$ indicate the truss element numbers and the letters A-H denote the nodal points

$\mathbf{x}^{(m)}$ are empty. The 12 constrained deformations are collected in vector $\boldsymbol{\varepsilon}^{(o)}$ in the order of the element numbers shown in Fig. 2(a). The dependent coordinates $\mathbf{x}^{(c)}$ of the nodal points are given in the left half of Table 2. For this system, (12) becomes

$$
\left[\begin{array}{cccccccccccc}
+\frac{1}{2} \sqrt{2} & 0 & +\frac{1}{2} \sqrt{2} & 0 & 0 & 0 & 0 & 0 & 0 & 0 & 0 & 0 \\
-\frac{1}{2} \sqrt{2} & 0 & +\frac{1}{2} \sqrt{2} & 0 & 0 & 0 & 0 & 0 & 0 & 0 & 0 & 0 \\
0 & 0 & 0 & 0 & +\frac{1}{2} \sqrt{2} & +\frac{1}{2} \sqrt{2} & 0 & 0 & 0 & 0 & 0 & 0 \\
0 & 0 & 0 & 0 & -\frac{1}{2} \sqrt{2} & +\frac{1}{2} \sqrt{2} & 0 & 0 & 0 & 0 & 0 & 0 \\
0 & 0 & 0 & 0 & 0 & 0 & -\frac{1}{2} \sqrt{2} & 0 & +\frac{1}{2} \sqrt{2} & 0 & 0 & 0 \\
0 & 0 & 0 & 0 & 0 & 0 & +\frac{1}{2} \sqrt{2} & 0 & +\frac{1}{2} \sqrt{2} & 0 & 0 & 0 \\
0 & 0 & 0 & 0 & 0 & 0 & 0 & 0 & 0 & 0 & -\frac{1}{2} \sqrt{2} & +\frac{1}{2} \sqrt{2} \\
0 & 0 & 0 & 0 & 0 & 0 & 0 & 0 & 0 & 0 & +\frac{1}{2} \sqrt{2} & +\frac{1}{2} \sqrt{2} \\
-\frac{1}{2} \sqrt{2} & -\frac{1}{2} \sqrt{2} & 0 & +\frac{1}{2} \sqrt{2} & +\frac{1}{2} \sqrt{2} & 0 & 0 & 0 & 0 & 0 & 0 & 0 \\
0 & 0 & 0 & +\frac{1}{2} \sqrt{2} & -\frac{1}{2} \sqrt{2} & 0 & -\frac{1}{2} \sqrt{2} & +\frac{1}{2} \sqrt{2} & 0 & 0 & 0 & 0 \\
0 & 0 & 0 & 0 & 0 & 0 & +\frac{1}{2} \sqrt{2} & +\frac{1}{2} \sqrt{2} & 0 & -\frac{1}{2} \sqrt{2} & -\frac{1}{2} \sqrt{2} & 0 \\
+\frac{1}{2} \sqrt{2} & -\frac{1}{2} \sqrt{2} & 0 & 0 & 0 & 0 & 0 & 0 & 0 & -\frac{1}{2} \sqrt{2} & +\frac{1}{2} \sqrt{2} & 0
\end{array}\right]
$$

$$
\times\left[\begin{array}{l}
\dot{x}^{E} \\
\dot{y}^{E} \\
\dot{z}^{E} \\
\dot{x}^{F} \\
\dot{y}^{F} \\
\dot{z}^{F} \\
\dot{x}^{G} \\
\dot{y}^{G} \\
\dot{z}^{G} \\
\dot{x}^{H} \\
\dot{y}^{H} \\
\dot{z}^{H}
\end{array}\right]=\left[\begin{array}{l}
\dot{\varepsilon}_{1}^{(1)} \\
\dot{\varepsilon}_{1}^{(2)} \\
\dot{\varepsilon}_{1}^{(3)} \\
\dot{\varepsilon}_{1}^{(4)} \\
\dot{\varepsilon}_{1}^{(5)} \\
\dot{\varepsilon}_{1}^{(6)} \\
\dot{\varepsilon}_{1}^{(7)} \\
\dot{\varepsilon}_{1}^{(8)} \\
\dot{\varepsilon}_{1}^{(9)} \\
\dot{\varepsilon}_{1}^{(10)} \\
\dot{\varepsilon}_{1}^{(11)} \\
\dot{\varepsilon}_{1}^{(12)}
\end{array}\right],
$$


Table 2 Dependent coordinates $\dot{\mathbf{x}}^{(c)}$ of the system with 12 rigid trusses, Fig. 2, and their kinematically indeterminate motion

\begin{tabular}{|c|c|c|}
\hline Node & Coordinates $\mathbf{x}^{(c)}$ & Velocity $\dot{\mathbf{x}}^{(c)}$ from $\mathbf{V}$ \\
\hline $\mathrm{E}$ & $(0,-1,1)$ & $\left(\begin{array}{l}0,-0.5,0) \\
0\end{array}\right.$ \\
\hline $\mathrm{F}$ & $\left(\begin{array}{lll}1, & 0, & 1\end{array}\right)$ & $(-0.5, \quad 0,0)$ \\
\hline G & $(0,1,1)$ & $\left(\begin{array}{lll}0, & 0.5, & 0\end{array}\right)$ \\
\hline $\mathrm{H}$ & $(-1, \quad 0,1)$ & $\left(\begin{array}{lll}0.5, & 0 & 0\end{array}\right)$ \\
\hline
\end{tabular}

in which the square $12 \times 12$ Jacobian matrix $\mathbf{D}^{(c)}$ can be recognised. The matrix clearly relates changes in the dependent nodal coordinates of nodes $\mathrm{E}-\mathrm{H}$ and elongations of the trusses.

The smallest singular value of matrix $\mathbf{D}^{(c)}$ appears to be zero indicating that the system has both one statically indeterminate mode and one kinematically indeterminate mode. The motion associated with the latter follows from the last column of the right singular matrix and is given in the right half of Table 2. Apparently, the square of the four trusses in the $z=1$ plane can deform as is visualised in Fig. 2(b). Note that the motion of the system is illustrated by assuming the velocity $\dot{\mathbf{x}}^{(c)}$ is applied for some finite time. The constraint equations for the elongations are then not necessarily satisfied. A more accurate analysis of the motion would require a non-linear simulation which is not needed to illustrate the motion of this unconstrained mode.

The last column of the left singular matrix $\mathbf{U}$ represents the statically indeterminate stress resultants, which are the longitudinal stress resultants (normal forces) in the trusses. All values are non-zero and equal to $\pm \frac{1}{6} \sqrt{3}$, where half of the stress resultants show a negative sign and the other half are positive. The graph in Fig. 2(a) shows these stress resultants where the different colours indicate the positive and negative stress resultants. The overconstrained condition can be avoided by allowing the elongation in one of the trusses. Note that the exact value of the stress resultants does not have a physical meaning as the vector with the stress resultants can be multiplied by any positive or negative constant to obtain another set of stress resultants representing a statically indeterminate mode.

\section{Exact constraint design of a compliant mechanism}

Next, it will be shown how the proposed analysis can be applied for the exact constraint design of a compliant mechanism. A system is exactly constrained if it is both kinematically and statically determinate. According to Table 1 , this implies that $\mathbf{D}^{(c)}$ should be square $(m=n)$ and non-singular. This kinematic exact constraint design approach is detailed in this section. As an example a straight guidance mechanism is considered in which some rigid part should move in one translational direction while all other motions must be suppressed.

\subsection{Straight guidance mechanism}

A typical approach for the design of the straight guidance mechanism is to assemble it from parts that confine one or more degrees of freedom each. To achieve an exact constraint design, no degree of freedom should be confined more than once and the desired translational motion of the system should not be constrained. In the concept of Fig. 3(a) five wire flexures are applied. The longitudinal stiffness of the wire flexure is relatively high, while the stiffnesses for bending and torsion are rather small. So, each wire flexure restricts only one degree of freedom being the translation along its longitudinal axis. The straight guidance 


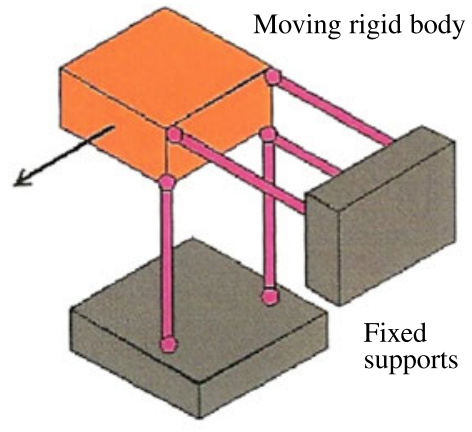

(a) five wire flexures

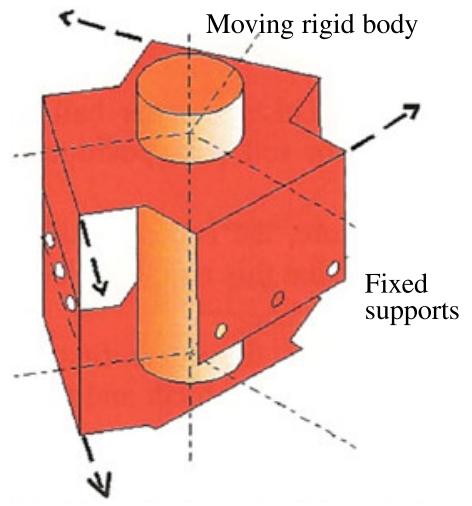

(b) five folded sheet flexures

Fig. 3 Two concepts for a one degree-of-freedom (1-DOF) straight guiding mechanism (reprinted from [15]). In (a), the solid arrow indicates the free movement. In (b), the cylinder represents the vertically moving part. The supports are at the holes. The dashed arrows indicate directions of constrained movements. The fifth folded sheet flexure at the lower back of the mechanism is hardly visible and its constraint is not shown in the figure

can be achieved with any combination of five wire flexures that are not aligned with the direction of the intended motion and that do not confine the same degree of freedom more than once.

A folded sheet flexure also restricts one degree of freedom as will be detailed in the next subsection. So, five folded sheet flexures can also be used for the straight guidance as shown in Fig. 3(b). The cylindrical rigid body is attached to all five folded sheet flexures, thus leaving only one degree of freedom for the motion of the cylinder if the flexures are aligned correctly.

\subsection{Kinematic analysis of wire flexures and folded sheet flexures}

For the kinematic analysis of a system, the compliant parts are modelled such that the elastic deformations with low stiffness are allowed to vary, whereas the other deformations are suppressed. In the system with five wire flexures, Fig. 3(a), each of the wire flexure supports is modelled with one beam element. One end of the beam is fully supported, which means that all translational and rotational coordinates are fixed $\left(\mathbf{x}^{(o)}\right)$. The wire flexure allows bending in either direction as well as torsion around its longitudinal axis. The relatively large longitudinal stiffness disallows elongation $\varepsilon_{1}$ of the element. So, there is one constrained deformation $\left(\varepsilon^{(o)}\right)$ in each element and the other five deformations are defined to be dependent $\left(\varepsilon^{(c)}\right)$. For the coordinates at the free end of the wire flexure, one constraint equation must be satisfied leaving five independent coordinates.

In the system of Fig. 3(a), there are five wire flexures that are combined to define the motion of one rigid body. As each wire flexure adds one constraint for the translational and rotational coordinates of the motion of its free end, the combination of all five wire flexures leaves one degree of freedom if the constraints are independent. Matrix $\mathbf{D}^{(c)}$ must be square as can be verified easily. The number of rows equals the number of constrained and independent deformations. In each wire flexure, the elongation is fixed, so there are five rows. The number of columns equals the number of dependent coordinates. These coordinates are translational and rotational coordinates of the moving rigid body. If the intended translation 


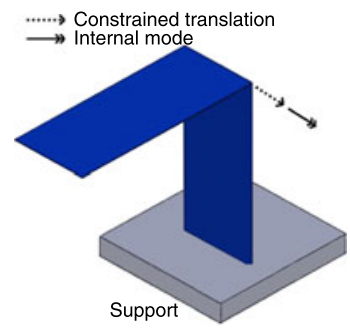

(a) single folded sheet flexure support

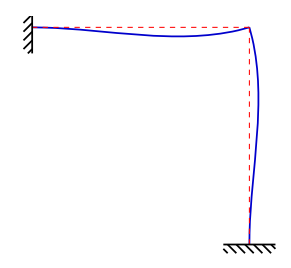

(b) internal deformation mode (side view)

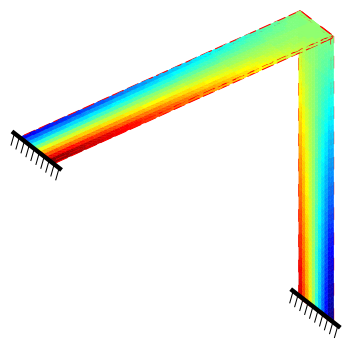

(c) statically indeterminate bending stress distribution with both ends fixed (blue and red indicate opposite sign of the stresses)

Fig. 4 Constrained translation and internal mode of a single folded sheet flexure

is defined as the independent coordinate $x^{(m)}$, then five dependent coordinates remain and $\mathbf{D}^{(c)}$ is indeed square.

Modelling the system with the folded sheet flexures, Fig. 3(b), is somewhat more complicated. A support with a folded sheet flexure as shown in Fig. 4(a) consists of two sheet flexures in series. As was already mentioned in Sect. 2.1, out-of-plane bending and torsion are allowed for each sheet flexure while the stiffnesses for bending in the plane of the sheet and elongation are high. For the kinematic analysis, one beam element is used for each sheet in the flexure. Taking the local $z$-axis of the beam element in the plane of the sheet as illustrated in Fig. 1, the constrained deformations are the elongation $\varepsilon_{1}$ and the bending deformations $\varepsilon_{3,4}$ in the in-plane $z$-direction. The torsion $\varepsilon_{2}$ and bending deformations $\varepsilon_{5,6}$ in the out-of-plane $y$-direction are the other three deformations that are allowed to vary. Combining two sheets, there are six deformations in the folded sheet flexure that are not suppressed. It is not correct to define all these deformations to be dependent deformations as that would suggest that there are no constraints for the six coordinates of the free end. As shown in Fig. 4(a), the folded sheet flexure imposes one constraint being a translation of the connection line between the sheets. There is also an internal deformation mode, which is a rotation around the axis of this connection line. Figure 4(b) illustrates that this internal mode can occur even if both ends of the folded sheet flexure are fixed. This double clamped folded sheet flexure has a statically indeterminate stress distribution as well. This is illustrated in Fig. 4(c) where the bending stress distribution in the beams is shown. At the clamped ends, the stresses have opposite signs which indicates that the over-constraint is due to this in-plane bending mode.

The constrained and internal modes of the folded sheet flexure can be accounted for by taking one of the bending deformations as an independent deformation, thus leaving five dependent deformations. In this way for the coordinates at the free end of the folded sheet flexure, one constraint equation has to be satisfied leaving five independent coordinates similar as for a single wire flexure. In addition, each folded sheet flexure exhibits one internal independent deformation mode.

Combining five folded sheet flexures as in the system of Fig. 3(b) leaves one independent coordinate for the cylindrical rigid body to which all flexures are attached, if the five constraints are independent. Also in this case, matrix $\mathbf{D}^{(c)}$ must be square. In each folded sheet flexure, there are six constrained deformations and one independent deformation, so there are 35 rows in total. Counting the dependent coordinates, we observe that in each flexure 


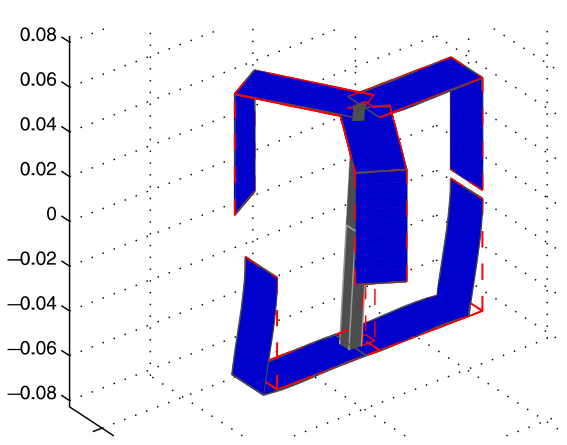

(a) Kinematically indeterminate motion

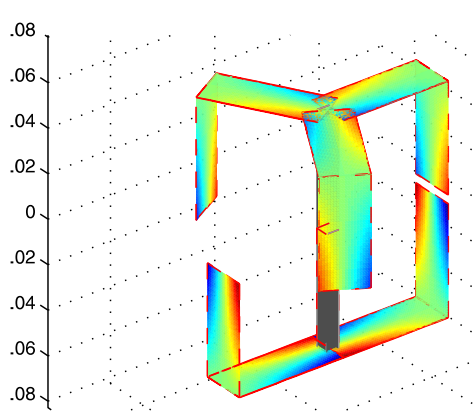

(b) Statically indeterminate bending stress distribution (blue and red indicate opposite sign of the stresses)

Fig. 5 Alternative straight guiding mechanism: An over-constrained and under-constrained design with five folded sheet flexures. The fixed supports are at the five unconnected ends of the folded sheet flexures

the six coordinates of translation and rotation of the connection between the sheets are dependent. Furthermore, the rigid connection of all flexures has one independent coordinate, leaving five dependent coordinates. So, $\mathbf{D}^{(c)}$ has 35 columns and is indeed square. It can be verified numerically that $\mathbf{D}^{(c)}$ is non-singular for the configuration shown in Fig. 3(b). The actual size of matrix $\mathbf{D}^{(c)}$ may be larger if there are more nodal points used to model the translating rigid part. As this part is rigid, there is also a constrained deformation for every extra nodal coordinate, so $\mathbf{D}^{(c)}$ remains square.

A drawback of the design in Fig. 3(b) is its asymmetry. A symmetric design can offer advantages, e.g. to reduce position errors of the moving rigid part due to temperature changes. Furthermore, two folded flexures on one side offer a lower support stiffness compared with three folded flexures on the other side.

A more symmetric design is presented in Fig. 5. With five folded sheet flexures, the symmetry at the lower end is improved by changing the position and orientation of one of the folded sheet flexures. However, this does not increase the support stiffness, and even worse, a kinematic analysis reveals immediately that this design is both under-constrained and over-constrained. As the constrained degrees of freedom of both lower flexures are parallel, these constraints are not independent, thus allowing the kinematically indeterminate motion shown in Fig. 5(a). The statically indeterminate bending stress distribution in Fig. 5(b) is similar to Fig. 4(c) from which it is concluded that the over-constraint is due to the constrained in-plane bending mode of the sheets.

With six-folded sheet flexures, the symmetric design of Fig. 6 is realised. As the extra flexure adds an extra constraint, this design is over-constrained. The statically indeterminate stress distribution shown in the figure indicates an in-plane bending stress in all sheet flexures, again similar to the single flexure in Fig. 4(c). At this stage, the designer can decide to continue with the over-constrained design, accepting the risk that this may cause unwanted behaviour, e.g. caused by misalignments due to manufacturing inaccuracies [13]. If an exact constraint design must be realised, this can be accomplished by allowing a bending motion in one of the sheets or its support. Alternatively, a purely torsional deformation in the rigid body can be released. 
Fig. 6 Alternative straight guiding mechanism: An over-constrained design with six folded sheet flexures. The fixed supports are at the six unconnected ends of the folded sheet flexures

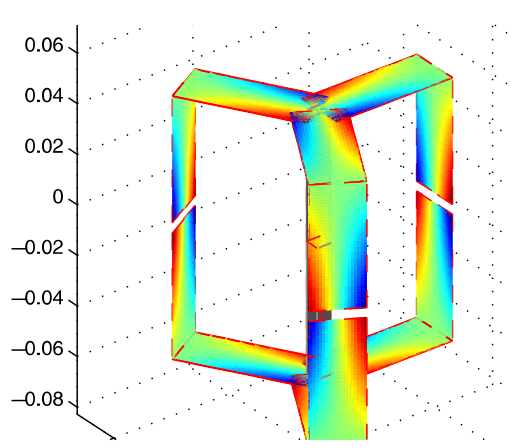

Statically indeterminate bending stress distribution (blue and red indicate opposite sign of the stresses)

\section{Conclusions}

This paper has demonstrated that a finite element based multibody dynamics methodology can be used effectively to analyse the constraints and degrees of freedom in compliant mechanisms. In the kinematic analysis, all deformations associated with a high support stiffness are taken to be invariant. Based on a clear notion of the system's relative and absolute constraints as well as the degrees of freedom, the Jacobian matrix associated with the dependent coordinates $\mathbf{D}^{(c)}$ is established. Rank deficiency of this matrix is detected using a singular value decomposition. Column rank deficiency indicates that the system is under-constrained. The kinematically indeterminate modes are found in the right singular matrix and can directly be used to visualise the motion associated with these under-constrained modes. Row rank deficiency of the Jacobian matrix $\mathbf{D}^{(c)}$ indicates an over-constrained system. In this case, one or more statically indeterminate stress distributions are derived from generalised stress resultants in the left singular matrix and are used to visualise the over-constraints. In this way, the presented analysis and visualisation not only detect under-constrained and over-constrained conditions, but also offer insight in the nature of the under-constrained and over-constrained modes. In this paper, the approach is demonstrated with straight guiding mechanisms. It paves the path toward a systematic approach to realise systems satisfying exact constraint design [6].

Acknowledgements The authors acknowledge the contributions from Steven Boer and Tjeerd van der Poel to the postprocessing and visualisation.

Open Access This article is distributed under the terms of the Creative Commons Attribution Noncommercial License which permits any noncommercial use, distribution, and reproduction in any medium, provided the original author(s) and source are credited.

\section{References}

1. Angeles, J., Gosselin, C.: Détermination du degré de liberté des chaînes cinématiques. Trans. Can. Soc. Mech. Eng. 12(4), 219-226 (1988)

2. Besseling, J.F.: Finite element methods. In: Besseling J., van der Heijden A. (eds.) Trends in Solid Mechanics-Proceedings of the Symposium Dedicated to the 65th Birthday of W.T. Koiter, pp. 53-78. Delft University Press, Delft (1979)

3. Blanding, D.L.: Exact Constraint: Machine Design Using Kinematic Principles. ASME Press, New York (1999) 
4. Boer, S.E., Aarts, R.G.K.M., Brouwer, D.M., Jonker, J.B.: Multibody modelling and optimization of a curved hinge flexure. In: The 1st Joint International Conference on Multibody System Dynamics, Lappeenranta, Finland (2010)

5. Bottema, O.: On Grübler's formulae for mechanisms. Appl. Sci. Res., Sect. A A2, 162-164 (1951)

6. Aarts, R.G.K.M., Boer, S.E., Meijaard, J.P., Brouwer, D.M., Jonker, J.B.: Analyzing overconstrained design of compliant mechanisms. Paper no. DETC2011-48548. In: Proceedings of the ASME 2011 International Design Engineering Technical Conferences \& Computers and Information in Engineering Conference (IDETC/CIE 2011), Washington, DC, USA, August 28-31, 2011 (2011)

7. Golub, G.H., Reinsch, C.: Singular value decomposition and least squares solutions. Numer. Math. 14(5), 403-420 (1970)

8. Grübler, Martin: Allgemeine Eigenschaften der zwangläufigen ebenen kinematischen Ketten. Der Civil. 29, 167-200 (1883)

9. Jonker, J.B.: A finite element dynamic analysis of flexible spatial mechanisms and manipulators. Comput. Methods Appl. Mech. Eng. 76, 17-40 (1989)

10. Jonker, J.B., Meijaard, J.P.: SPACAR - computer program for dynamic analysis of flexible spatial mechanisms and manipulators. In: Schiehlen W. (ed.) Multibody Systems Handbook, pp. 123-143. Springer, Berlin (1990)

11. Jonker, J.B., Meijaard, J.P.: Definition of deformation parameters for the beam element and their use in flexible multibody system analysis. In: ECCOMAS Thematic Conference Multibody Dynamics 2009. Warsaw University of Technology, Warsaw (2009)

12. Jonker, J.B., Aarts, R.G.K.M., van Dijk, J.: A linearized input-output representation of flexible multibody systems for control synthesis. Multibody Syst. Dyn. 21(2), 99-122 (2009)

13. Meijaard, J.P., Brouwer, D.M., Jonker, J.B.: Analytical and experimental investigation of a parallel leaf spring guidance. Multibody Syst. Dyn. 23(1), 77-97 (2010)

14. Pellegrino, S., Calladine C.R.: Matrix analysis of statically and kinematically indeterminate frameworks. Int. J. Solids Struct. 22(4), 409-428 (1986)

15. Soemers, H.M.J.R.: Design Principles for Precision Mechanisms. T-point Print, Enschede (2010) 